\title{
(QUARK) ONIUM THEORY AND SPECTROSCOPY
}

\author{
C. Quigg \\ Fermi National Accelerator Laboratory \\ Batavia, Illinois 60510
}

\begin{abstract}
Introductory remarks to Parallel Session $\mathrm{C7}$ at the Xxth International Conference on High Energy Physics.
\end{abstract}

Now, nearly six years after the discovery of charmonium, the nonrelativistic theory of quarkonium spectroscopy has achieved a degree of maturity and permanence. For the most part, therefore, the contributions made to the subject in the past year represent extensions and refinements of calculations or analyses of new data, rather than novel theoretical initiatives.

The extensive results on the vector states in the $T$ family obtained at CESR, together with new measurements of $T$ and ${ }^{\prime}$ ' properties at DORIS, make it possible to further the program of unbiased (or "model-independent") determinations of the interquark interaction. Two facets of this line of investigation are reviewed in this session. What may be called the elementary quantum mechanics approach, in which the dependence of observables upon quark mass or principal quantum number is used to infer properties of the potential, is discussed in the contribution by André Miartin. A complementary program in which bound-state properties are used to reconstruct the potential by means of inverse scattering techniques is the subject of Jonathan Rosner's report. Both methods provide support for the idea that the quark-antiquark interaction is flavor-independent. No direct evidence has been found for the one-gluonexchange coulomb potential at short distances; it appears that this awaits heavier quarkonia.

A key aspect of the nonrelativistic treatment is the need to make precise connections between properties of the Schrödinger wavefunction and observables. At issue are the importance of relativistic effects and of radiative (which is to say higher-order QCD) corrections to transition rates. Transcription of QED radiative correction formulae has led to the suspicion that such corrections may be large for quarkonium decays. Recent work, summarized here by W. Celmaster, suggests that at least for certain ratios of decay rates the corrections may not be uncontrollably large. The reliability of lowest-order expressions for $a b-$ solute rates is still not established. 
A worthy goal is the derivation of the interquark potential from a fundamental theory. Considerations of this sort lead to the expectations of coulomb and linear potentials at very short and very long distances, and have yielded interesting suggestions for interpolations in the region of current experimental concern. QCD-inspired ruminations also point to the likelihood that non-quark degrees of freedom may manifest themselves as "extra" levels which cannot be reproduced by potential models. These may be

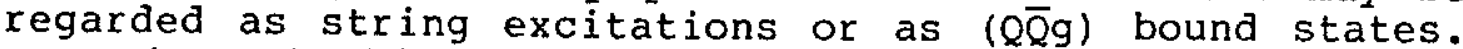
One view of this situation from the perspective of the MIT/Budapest bag model is presented by Julius Kuti.

Several topics on which there has been recent progress reported in contributions to this Conference could not be included in the presentations to this session. These include new work on hadronic transitions among quarkonium states, the influence of flavor threshold on upsilon line shapes, and theorems on bound-state properties. The Kepler problem of light quark-heavy quark bound states and extensions of sum rules for quarkonium states have also been omitted.

A final contribution to this, session, by S. Meshkov, deals with quarkonium as an entrée to other new hadronic states. The specific issue addressed is whether the $\mathrm{E}(1440)$ meson observed in the decay $\psi \rightarrow \gamma+$ anything is to be interpreted as a glueball state in the process $\psi \rightarrow \gamma+(\mathrm{gg})$. More extended discussions of the characteristics of glueballs (gluonia?) have been presented by John Donoghue in parallel session A2 and by Carl Carlson in session Dlo. 